\title{
Relating Casimir to Magnetic Energies Results in Spatial Dimensions That Define Biology Systems
}

\author{
Michael A. Persinger \\ Laurentian University, Sudbury, Ontario P3E 2C6, Canada \\ E-mail address: mpersinger@laurentian.ca
}

\begin{abstract}
The volume-independence that occurs when Casimir and magnetic energies were equated was employed to solve for optimal spatial separations. For the magnetic moments of a proton and an electron in the presence of a magnetic field strength that produced the energy associated with the neutral hydrogen line, the distances were $1 \mathrm{~nm}$ and $24 \mathrm{~nm}$ or the width of an ion channel in a plasma cell membrane and the average synaptic width, respectively. The small discrepancies in orbit-spin magnetic moments of the electron with the magnetic moment of the proton emerged as relevant. Calculation of the radius in the bound (circular) system associated with the required magnetic field strength for the $\sim 3.41 \cdot 10^{-27} \mathrm{~A} \cdot \mathrm{m}^{2}$ discrepancy solved as the Compton wavelength of the electron. Applications of the approach allowed quantitative convergence between universal photon densities within $1 \mathrm{~nm}$ widths as well as integration of the energy from acceleration for estimated upper limits of resting photon masses with Planck's constant. The results suggest that the physical and chemical properties that define biological systems, particularly the brain, reflect astronomical principles.
\end{abstract}

Keywords: Casimir energy; magnetic energy; neutral hydrogen line energy; magnetic moments; photon rest mass; emergent properties

\section{INTRODUCTION}

One approach to provide a unified perspective of the universe is to integrate the most fundamental and inferred recondite variables that define our perception of the boundaries of space and time within the human brain [1]. The implicit procedure is to relate energies which, if Helmholtz is correct, reflect an intrinsic unity manifested in multiple forms that should reveal the general nature of and the relationship between the different levels of scientific discourse. They define astronomy, physics, chemistry, biology and the behavioural sciences.

Levels of discourse are the arbitrary semantic designations for the increments of space and time in conjunction with their representations of matter (structure) and energy (dynamics) of the Natural World. The relationship between Casimir energy and magnetic energy reveals one possible relationship by which energies likely derived from vacuum fluctuations or a strong correlate could relate to the most fundamental spatial parameters within biological systems: the widths of the synapse and the ion channel. 


\section{RELATING CASIMIR TO MAGNETIC ENERGY}

If energy emerging from the Casimir force [2] is set equal to classic inferences of magnetic energy [3],

$$
\pi^{2}(240)^{-1} \hbar c m^{3}=B^{2}(2 \mu)^{-1} \mathrm{~m}^{3}
$$

where, $\mathrm{h}$ is the modified Planck's constant $\left(1.05 \cdot 10^{-34} \mathrm{~J} \cdot \mathrm{s}\right), \mathrm{c}$ is the velocity of light in a vacuum $\left(\sim 3 \cdot 10^{8} \mathrm{~m} \cdot \mathrm{s}^{-1}\right), \mu$ is the magnetic permeability $\left(1.26 \cdot 10^{-6} \mathrm{~N} \cdot \mathrm{A}^{-2}\right), \mathrm{m}^{3}$ is volume and $\mathrm{B}$ is the magnetic field strength and one solves for $\mathrm{B}$, volume $\left(\mathrm{m}^{3}\right)$ is no longer in the equation. The implications are significant because the relationship is potentially "scale invariant".

The resulting conditions allow an opportunity to discern the optimal distance between two neutral parallel plates or their spherical equivalents, such that:

$$
\mathrm{a}^{4}=\left[\pi^{2}(240)^{-1} \cdot \hbar c \cdot 2 \mu\right] \cdot \mathrm{B}^{-2}
$$

The only variable in this equation would be magnetic field strength. The value of the numerator is fixed. My approach is that one primary unifier or integrator of universal or astronomical forces applied to local physics and chemistry within biological spaces is the hydrogen line or $1.42 \cdot 10^{9} \mathrm{~Hz}(1.42 \mathrm{GHz})$. The energy associated with this frequency, obtained by multiplication with Planck's constant $\left(6.626 \cdot 10^{-34} \mathrm{~J} \cdot \mathrm{s}\right)$, is $9.41 \cdot 10^{-25} \mathrm{~J}$.

The magnetic moment of the circulating current created by an electron moving in its closed path is $9.274 \cdot 10^{-24} \mathrm{~A} \cdot \mathrm{m}^{2}\left(\mathrm{~J} \cdot \mathrm{T}^{-1}\right)$. Hence the required magnetic field strength would be the ratio or $1.01 \cdot 10^{-1} \mathrm{~T}$. If the square of that value $\left(1.02 \cdot 10^{-2} \mathrm{~T}^{2}\right)$ is inserted into equation $(2)$, the optimal separation between ideal parallel plates would be $\sim 24 \mathrm{~nm}$. Application of the central limit theorem and assuming the typical standard deviations of $\sim 30 \%$ of the central tendency, the range for $95 \%$ of the spaces would be between 10 and $37 \mathrm{~nm}$. This is the range of the width of a synapse, the interface between neurons [4].

On the other hand if the magnetic moment of the proton $\left(1.41 \cdot 10^{-26} \mathrm{~A} \cdot \mathrm{m}^{2}\right)$ is employed, the magnetic field strength required to obtain the energy associated with the hydrogen line is $6.67 \cdot 10^{1} \mathrm{~T}$. If this value squared $\left(44.5 \cdot 10^{2} \mathrm{~T}^{2}\right)$ is inserted into equation (2), the resulting width (a) is $0.93 \cdot 10^{-9} \mathrm{~m}$. This is within the median range of the width of an ion channel found in plasma cell membranes [5]. Most "channels" within the plasma cell membrane are those that mediate the movements of protons [6].

According to previous calculations [7] regarding the mass estimates of the universe and the upper limit for the rest mass of the photon there would be $\sim 10^{104}$ rest mass photon equivalents for the universal mass $\left(\sim 10^{52} \mathrm{~kg}\right)$ contained within an estimated volume of between $10^{78}$ and $10^{79} \mathrm{~m}^{3}$ depending upon the progression towards the final epoch. The averaged density would be between $10^{-26}$ to $10^{-27} \mathrm{~m}^{3}$ per photon unit or a functional length of $\sim 10^{-9} \mathrm{~m}$, the width a channel.

From this perspective the relationship between the times required for an electron to complete one orbit in a Bohr atom and the velocity of light become directly applicable. For a photon displaying a vacuum velocity or its mild modifications in matter such as water $\left(2.8 \cdot 10^{8} \mathrm{~m} \cdot \mathrm{s}^{-1}\right)$, the time required to traverse a plasma cell membrane $\left(\sim 10^{-8} \mathrm{~m}\right)$ would be $\sim 10^{-}$ $16 \mathrm{~s}$. Such temporal juxtaposition could allow an interaction, such as the potential superimposition of information, between the photon and the value for the single electron orbit which is a component of the basis of Planck's constant. 
This relationship is not trivial. One-half of the mass $\left(9.11 \cdot 10^{-31} \mathrm{~kg}\right)$ of an electron multiplied by the square of the fine-structure velocity $\left(4.8 \cdot 10^{12} \mathrm{~m}^{2} \cdot \mathrm{s}^{-2}\right)$ results in a fixed increment of energy of $\sim 43.7 \cdot 10^{-19} \mathrm{~J}$. The time required for one orbit of an electron in a Bohr atom is $\sim 1.5 \cdot 10^{-16} \mathrm{~s}$. The resulting value is $\sim 6.6 \cdot 10^{-34} \mathrm{~J} \cdot \mathrm{s}$, or within measurement error of the classical Planck's constant.

If there were a dimensional transform for this "photon" unit within the dimensions of a plasma cell membrane channel as some essential feature of space, the results would be revealing. The energy associated with a photon at the currently [8] accepted upper limit of the rest mass is $\leq 4 \cdot 10^{-52} \mathrm{~kg}$. If we assume (n-1) or $3 \cdot 10^{-52} \mathrm{~kg}$, for convenience, as the threshold oscillatory value and it were accelerated to $\mathrm{c}\left(9 \cdot 10^{16} \mathrm{~m}^{2} \cdot \mathrm{s}^{-2}\right)$ the emergent energy is $2.7 \cdot 10^{-35} \mathrm{~J}$. However this is effectively the same value as this rest mass moving at the velocity light with a vibration of the neutral hydrogen line $\left(1.42 \cdot 10^{9} \mathrm{~Hz}\right)$ across the $21 \mathrm{~cm}$ wavelength.

Compared to a unit $\mathrm{Hz}$ for Planck's constant the energy from accelerating a photon with this upper limit of rest mass is about $8 \pi$ less. For the value to be exactly $8 \pi$, the upper limit of the rest mass of a photon must be $2.93 \cdot 10^{-52} \mathrm{~kg}$. The involvement of this parameter reiterates the potential connection between components associated with cosmological constants and local phenomena. It also suggests that the second derivative of the rate of change of the surface of a sphere may help relate the shared origin of the photon and the electron.

Although more precise instrumentation will be required to verify this prediction, the implications that the photon displays a rest mass allows the emergence of a third state of polarization (where the vector of the electric field points along the line of motion), the phase shifting of electrons diffracting around a tubular magnetic flux (such as an ion channel) due to the presence of the vector potential $\mathbf{A}$, and the direct influence of the Casimir phenomena upon massive photons [8].

\section{APPLICATIONS}

If a proton was moving through a channel with a radius of $0.93 \cdot 10^{-9} \mathrm{~m}$, according to the more classic equation:

$$
B=\mu_{o} I(2 \pi r)^{-1}
$$

where $\mu_{\mathrm{o}}$ is the magnetic permeability and $\mathrm{I}$ is the current, in this case a unit charge $\left(1.6 \cdot 10^{-19}\right.$ $\mathrm{A} \cdot \mathrm{s})$, the value would be $0.35 \cdot 10^{-16} \mathrm{~kg} \cdot \mathrm{A}^{-1} \mathrm{~s}^{-1}$. When this value is multiplied by the hydrogen line $1.42 \cdot 10^{9} \mathrm{~s}^{-1}$, the magnetic field strength is $4.9 \cdot 10^{-8} \mathrm{~T}$ or $\sim 50 \mathrm{nT}$.

This value is within error measurement of the estimated average magnetic energy of $\sim 25 \mathrm{nT}$ [9] associated with the total energy of the universe within the current volume. This value was derived from assumptions of the total mass of the universe being $2.4 \cdot 10^{52} \mathrm{~kg}$, the radius of $3.56 \cdot 10^{25} \mathrm{~m}$ and the total energy being $1.08 \cdot 10^{69} \mathrm{~J}$ which is also the kinetic energy of the Hamiltonian value representing the entire system.

If the frequency associated with $6.67 \cdot 10^{1} \mathrm{~T}$, the value required to obtain the width of an ion channel from the proton's magnetic moment, were derived the value would be $\left[\left(6.67 \cdot 10^{1}\right)\right.$ $\mathrm{T}] \cdot\left[0.35 \cdot 10^{-16} \mathrm{~kg} \cdot \mathrm{A}^{-1} \mathrm{~s}^{-1}\right]^{-1}$ or $19.05 \cdot 10^{17} \mathrm{~Hz}$. When divided into the velocity of light in a vacuum the $\lambda=1.6 \cdot 10^{-10} \mathrm{~m}$ which is almost one-half $(1 / 2)$ of the Bohr circumference. The potential significance for quantum numbers for relating gravitational-electromagnetic energy has been discussed elsewhere [10]. 


\section{EMERGENT PROPERTIES}

The radiative correction to the magnetic interaction with the energy of an electron corresponds to the additional magnetic moment associated with the electron's spin. The value is 1.00116 (often expressed as $0.00116,[11]$ ) is obtained by dividing the spin magnetic moment by the orbital moment. However the difference in magnetic moment of the Bohr magneton due to the orbit of an electron $\left(9.2740780 \mathrm{~A} \cdot \mathrm{m}^{2}\right)$ and the electron's spin $(9.2847701$ $\left.\mathrm{A} \cdot \mathrm{m}^{2}\right)$ is $1.0692 \cdot 10^{-26} \mathrm{~A} \cdot \mathrm{m}^{2}$. This value is very similar to the magnetic moment of the proton $\left(1.410617 \cdot 10^{-26} \mathrm{~A} \cdot \mathrm{m}^{2}\right)$ with a difference of $3.4141 \cdot 10^{-27} \mathrm{~A} \cdot \mathrm{m}^{2}$. The magnetic field strength required for this mild difference or discrepancy to match the neutral hydrogen line would be $2.76 \cdot 10^{2} \mathrm{~T}$.

Current is the product of unit charge and frequency in this context. For a unit charge $\left(1.6 \cdot 10^{-19} \mathrm{~A} \cdot \mathrm{s}\right)$ and the Bohr frequency $\left(6.58 \cdot 10^{15} \mathrm{~s}^{-1}\right)$ the current would be $1.05 \cdot 10^{-3} \mathrm{~A}$.

The absolute distance associated with a specific magnetic field strength is:

$$
\mathrm{R}=\mu_{\mathrm{o}} \mathrm{I}(2 \mathrm{~B})^{-1}
$$

and for a field strength of $2.76 \cdot 10^{2} \mathrm{~T}$, the distance is $2.4 \cdot 10^{-12} \mathrm{~m}$. This is the Compton wavelength of the electron. As expected from classical approaches, the frequency equivalence at $\mathrm{c}$ is $1.25 \cdot 10^{20} \mathrm{~Hz}$ that exhibits an energy (when multiplied by Planck's constant) of $8.28 \cdot 10^{-}$ ${ }^{14} \mathrm{~J}$. When divided by the square of the velocity of light $\left(9 \cdot 10^{16} \mathrm{~m}^{2} \cdot \mathrm{s}^{-2}\right)$ the quotient is within calculation error of the mass of the electron.

In other words the small discrepancy that has been known for decades between the magnetic moment for the spin-orbit difference for an electron and the magnetic moment of a proton could reflect a universal connection. Only when this diminutive increment of magnetic moment is multiplied by a magnetic field intensity that would allow the convergence with the energy of the neutral hydrogen line does the resulting distance merge with the wave form of the electron: the Compton wavelength.

Such a large magnetic field could appear to be non-rational. However such a field if it occurred transiently would be associated with energy. For the energy of the mass equivalence of an electron $\left(8.28 \cdot 10^{-14} \mathrm{~J}\right)$ to occur according to the right component of equation 1 , the volume would be $2.72 \cdot 10^{-24} \mathrm{~m}^{3}$ or the linear radius of $14 \mathrm{~nm}$ or the diameter of $28 \mathrm{~nm}$. This is the same functional distance as the area within which Casimir-electromagnetic energies would interact when the angular momentum of the (orbital) electron is solved for energies required from the hydrogen line.

\section{COSMIC BACKGROUND INFLUENCE}

If the changing magnetic field boundary [2] is required for a virtual particle existing within cosmic background to be transformed to a real particle the locus of this transformation would be expected to occur within the energies associated with the unit oscillation of matter. This too can be approximated by the Bohr atom.

The calculation of the equivalent magnetic field of the Bohr magneton from (3) for a unit charge moving around a circumference with the radius $=5.2 \cdot 10^{-11} \mathrm{~m}$ is $0.062 \cdot 10^{-14} \mathrm{~kg} \cdot \mathrm{A}^{-}$ ${ }^{1} \mathrm{~s}^{-1}$. When multiplied by the frequency of this orbital movement $\left(6.59 \cdot 10^{15} \mathrm{~s}^{-1}\right)$ the value is $\sim 4.0 \mathrm{~T}$. 
For the magnetic moment of the electron $\left(9.274 \cdot 10^{-24} \mathrm{~J} \cdot \mathrm{T}^{-1}\right)$ the resulting energy is $3.71 \cdot 10^{-23} \mathrm{~J}$ and when divided by the unit charge value is $2.3 \cdot 10^{-4} \mathrm{~V}$ or $0.23 \mathrm{mV}$. This increment of potential difference is within the root-mean squared measurement values (to accommodate polarity $\pm 0.23 \mathrm{mV}$ ) associated with the release of the contents of one average vesicle [4] containing 3 to 5 thousands of molecules form the presynaptic area. The vesicle has been considered a primary "quantum" associated with the transmission of chemicallybased information through the nervous system.

The value becomes relevant when the equivalent temperature is calculated. The energy $3.71 \cdot 10^{-23} \mathrm{~J}$ divided by the Boltzmann constant $\left(1.38 \cdot 10^{-23} \mathrm{~J} \cdot \mathrm{K}^{-1}\right.$ where $\mathrm{K}$ is the temperature in Kelvin, is the value $2.74^{\circ} \mathrm{K}$ or effectively the temperature of the cosmic background. This could suggest that the information within the cosmic background could influence the physical dynamics of vesicles that display a very precise shift in voltage upon the release of their contents into the synaptic cleft.

For comparison the increment of voltage associated with the opening of a single channel in a plasma cell membrane is $\sim 0.3 \mu \mathrm{V}$ [4] and the energy on a unit charge would be $4.8 \cdot 10^{-26} \mathrm{~J}$ or the equivalent of $3.48 \cdot 10^{-3}{ }^{\circ} \mathrm{K}$. This has the potential to be within the range of a functional "Einstein-Bose condensate". If this is correct then what is measured at the level of the individual ion channel should be reflected in the macrostructural physical characteristics of the brain [12].

\section{CONCLUSIONS}

The spatial dimensions that define the synapse and the ion channel were evident when the energies that converged Casimir and traditional magnetic forms were equated. The specific values required the presence of a magnetic field strength that when multiplied by the magnetic moment of the proton or of the electron resulted in the energy of the neutral hydrogen line. Subtle differences in the magnetic moments for the electrons spin and orbit, the proton's value and the slight discrepancies revealed features that could connect fundamental features of astronomical phenomena with those that contribute to quantum processes in the physical and chemical systems that define the brain.

\section{References}

[1] M. A. Persinger, International Letters of Chemistry, Physics and Astronomy 8 (2013) 8-19.

[2] M. Bordag, U. Mohideen, V. M. Mostempanenko, Physics Reports 353 (2001) 1-205.

[3] D. Halliday, R. Resnick, Physics: Combined Edition John Wiley and Sons, New York, 1962.

[4] E. R. Kandel, J. H. Schwartz, T. M. Jessell, Principles of Neural Science Eslevier, New York, 1991.

[5] G. M. Shepherd (ed), The Synaptic Organization of the Brain Oxford University Press, Oxford, 2004.

[6] T. E. DeCoursey, Physiological Reviews 83 (2002) 475-579.

[7] M. A. Persinger, The Open Astronomy Journal 5 (2012) 41-43. 
[8] C. T. Tu, J. Luo, G. T. Gilles, Reports of Progress in Physics 68 (2003) 77-130.

[9] M. A. Persinger, International Letters of Chemistry, Physics and Astronomy 11 (2014) $18-23$.

[10] M. A. Persinger, Neuroscience and Biobehavioral Reviews 36 (2012) 2334-2338.

[11] J. Schwinger, Physics Reviews 73 (1948) 416-418.

[12] M. A. Persinger, C. F. Lavallee, Journal of Consciousness Studies 19 (2012) 128-153. 